BNL-111886-2016-J A

\title{
Review of footnotes and annotations to the 1949- 2013 tables of standard atomic weights and tables of isotopic compositions of the elements (IUPAC Technical Report) 1
}

\author{
Tyler B. Coplen * and Norman E. Holden \\ *U.S. Geological Survey, Reston, Virginia, USA, \\ Brookhaven National Laboratory, Upton, New York, USA
}

Pure and Applied Chemistry

February 02, 2016

\author{
National Nuclear Data Center \\ Brookhaven National Laboratory \\ P.O. Box 5000 \\ Upton, NY 11973-5000 \\ www.nndc.bnl.gov
}

U.S. Department of Energy

Office of Science, Office of Nuclear Physics

\footnotetext{
Notice: This manuscript has been authored by employees of Brookhaven Science Associates, LLC under Contract No.DE-SC0012704 with the U.S. Department of Energy. The publisher by accepting the manuscript for publication acknowledges that the United States Government retains a non-exclusive, paid-up, irrevocable, world-wide license to publish or reproduce the published form of this manuscript, or allow others to do so, for United States Government purposes.
} 


\section{DISCLAIMER}

This report was prepared as an account of work sponsored by an agency of the United States Government. Neither the United States Government nor any agency thereof, nor any of their employees, nor any of their contractors, subcontractors, or their employees, makes any warranty, express or implied, or assumes any legal liability or responsibility for the accuracy, completeness, or any third party's use or the results of such use of any information, apparatus, product, or process disclosed, or represents that its use would not infringe privately owned rights. Reference herein to any specific commercial product, process, or service by trade name, trademark, manufacturer, or otherwise, does not necessarily constitute or imply its endorsement, recommendation, or favoring by the United States Government or any agency thereof or its contractors or subcontractors. The views and opinions of authors expressed herein do not necessarily state or reflect those of the United States Government or any agency thereof. 



\title{
IUPAC Technical Report
}

Tyler B. Coplen* and Norman E. Holden

\section{Review of footnotes and annotations to the 1949-2013 tables of standard atomic weights and tables of isotopic compositions of the elements (IUPAC Technical Report) ${ }^{1}$}

\begin{abstract}
The Commission on Isotopic Abundances and Atomic Weights uses annotations given in footnotes that are an integral part of the Tables of Standard Atomic Weights to alert users of the possibilities of quite extraordinary occurrences and sources with abnormal atomicweight values outside an otherwise acceptable range. The basic need for footnotes to the Standard Atomic Weights Table and equivalent annotations to the Table of Isotopic Compositions of the Elements arises from the necessity to provide to users information that is relevant to one or more elements, but that cannot be provided using numerical data in columns. Any desire to increase additional information conveyed by annotations to these Tables is tempered by the need to preserve a compact format and a style that can alert users, who would not be inclined to consult either the last full element-by-element review or the full text of a current Standard Atomic Weights of the Elements report.

Since 1989 the footnotes of the Tables of Standard Atomic Weights and the annotations in column 5 of the Table of Isotopic Compositions of the Elements have been harmonized by use of the three lowercase footnotes ("g”, “m”, and “ $r$ ") that signify geologically exceptionally specimens ("g”), modified isotopic compositions in material subjected to undisclosed or inadvertent isotopic fractionation ("m"), and the range in isotopic composition of normal terrestrial material prevents more precise atomic-weight value being given (“ $r$ ”). As elements are assigned intervals for their standard atomic-weight values (applies to 12 elements since 2009), footnotes "g" and " $r$ " are no longer needed for these elements.
\end{abstract}

Keywords: normal material; mononuclidic element; atomic weight variations; atomic weight intervals; Oklo natural reactor; stable isotope; undisclosed isotopic fractionation; artificial isotopic separation

\footnotetext{
${ }^{1}$ Sponsoring body: IUPAC Inorganic Chemistry Division: see more details on $\mathrm{p} . \mathrm{xxx}$

*Corresponding author: Tyler B. Coplen, U.S. Geological Survey, Reston, Virginia, USA, e-mail: tbcoplen@usgs.gov

Norman E. Holden, Brookhaven National Laboratory, Upton, New York, USA
} 


\section{Introduction}

From its inception in 1919, the International Union of Pure and Applied Chemistry (IUPAC) took over the careful evaluation and dissemination of atomic weights and their measurement uncertainties derived from critically assessed, published information. Recommended atomicweight values, $A_{\mathrm{r}}(\mathrm{E})$ of element $\mathrm{E}$, have been published under IUPAC since 1920. In 1979 [1], the Commission on Atomic Weights and Isotopic Abundances, as it was known then, agreed that an atomic weight could be defined for any specified sample and decreed that

"Dated Tables of Standard Atomic Weights published by the Commission refer to our best knowledge of the elements in natural terrestrial sources."

In recent times, the Table of Standard Atomic Weights (TSAW) has been published biennially, and their values are universally accepted throughout the world. Before 1979, the Commission Tables were titled "Table of Atomic Weights" or similarly, but the intent to publish tables of standard atomic weights was the same, and these pre-1979 tables are identified as TSAWs herein. Each standard atomic-weight value reflects the best knowledge of evaluated, published data $[2,3]$ of normal materials. The implied range of each standard atomic weight is intended to apply to all samples of sources of normal materials. In the Commission's 1969 report, a "normal" material was defined as [4]:

"A 'normal' material is one that contains as a major constituent a specified element with an atomic weight value that does not display a significant difference from the accepted value of that atomic weight because of:

(a) its radiogenic source;

(b) its extraterrestrial origin;

(c) artificial alteration;

(d) mutation; or

(e) a rare geological occurrence in small quantity."

This definition was updated in the 1971 TSAW [5] and "artificial alteration" was updated to "artificial isotopic fractionation" and "mutation" was updated to "artificial nuclear reaction". In 1984, the Commission revised the definition of a normal material to a material from a terrestrial source that satisfies the following criteria [2]:

"The material is a reasonably possible source for this element or its compounds in commerce, for industry or science; the material is not itself studied for some extraordinary anomaly and its isotopic composition has not been modified significantly in a geologically brief period."

This definition has been republished in several TSAWs, most recently in the 2013 TSAW [6]. For abnormal sources, an atomic-weight value may lie outside the normal range, and users are warned of such a possibility by footnotes to the TSAW. The use of such footnotes and annotations was summarized by Peiser et al. [2]:

"For quite extraordinary occurrences and other abnormal sources with abnormal atomic weights outside an otherwise acceptable range, the Commission uses annotations given in footnotes that are an integral part of the Tables of Standard Atomic Weights. Describing such abnormalities merely in the text of biennial reports would surely cause the warnings to 
be overlooked by more of the affected users. The detailed wording, not so much their intended meaning, has been modified from time to time.”

\section{Current footnotes and annotations of the Table of Standard Atomic Weights}

The 2013 TSAW [6] footnotes are reproduced here:

* Element has no stable isotopes. One or more representative isotopes are given in Table 4 with the appropriate relative atomic mass and half-life. However, four such elements ( $\mathrm{Bi}$, Th, $\mathrm{Pa}$, and $\mathrm{U}$ ) do have a characteristic terrestrial isotopic composition, and for these elements, standard atomic weights are tabulated.

g Geological materials are known in which the element has an isotopic composition outside the limits for normal material. The difference between the atomic weight of the element in such materials and that given in the table may exceed the stated uncertainty.

m Modified isotopic compositions may be found in commercially available material because the material has been subjected to an undisclosed or inadvertent isotopic fractionation. Substantial deviations in atomic weight of the element from that given in the table can occur.

$\mathrm{r}$ Range in isotopic composition of normal terrestrial material prevents a more precise standard-atomic weight being given; the tabulated value and uncertainty should be applicable to normal material.

A stable isotope is an isotope for which no radioactive decay has ever been experimentally measured. If a decay has been measured, the isotope is a radioactive isotope. The footnotes " $\mathrm{g}$ ", " $m$ ", and " $r$ " were selected intentionally because they are the first letter of the words "geological”, “modified”, and "range.”

The footnotes to the TSAW are linked directly to variations in isotopic composition of an element, which impacts it atomic weight. All known elements can be categorized according to the following constraints on their standard atomic weights [6,7]:

1. Elements with no stable isotope and with no radioactive isotope having a characteristic terrestrial isotopic composition in normal materials, e.g. radon. No standard atomic weight can be determined and no value is provided in the Table of Standard Atomic weights for these elements. These elements are assigned footnote asterisk $\left(^{*}\right)$.

2. Elements whose standard atomic weight is determined by only one stable isotope, e.g. sodium. The standard atomic weight is derived from the atomic mass of its stable isotope.

3. Elements whose standard atomic weight is determined by more than one isotope. They are subdivided into three groups:

a. Elements have no documented evidence of variation in atomic weight for normal materials, or elements that have not been evaluated for variation in isotopic composition by an IUPAC project, e.g., lanthanum. 
b. Elements have known variations in atomic weight in normal materials, but these variations do not exceed the evaluated measurement uncertainty of the atomic weight derived from the best measurement of the isotopic abundances of an element, e.g., molybdenum. Elements in this subcategory can advance to subcategory 3c as best-measurement of isotopic abundances improve.

c. Elements have known variations in atomic weight in normal materials that exceed the uncertainty of the atomic weight derived from a best measurement of isotopic abundances, but not yet assigned an atomic-weight interval by the Commission, e.g., copper. Elements in this subcategory can advance to category 4 as the Commission completes evaluations and assigns intervals. The Commission uses the footnote " $r$ " to identify elements in this subcategory for which the standard-atomicweight uncertainty has been expanded to account for known atomic-weight variability.

4. Elements with two or more isotopes having known variations in atomic weight in normal materials that exceed the uncertainty of the atomic weight derived from a best measurement of isotopic abundances and having upper and lower atomic-weight bounds determined by the Commission from evaluated, peer-reviewed, published data, e.g., carbon .

As discussed in the 2013 TSAW [6],

"The Commission uses the footnote "g" to identify chemical elements for which the recommended standard atomic weight and its associated uncertainty do not include all known variations. For example, some elements are anomalously enriched in fissionogenic or nucleogenic isotopes at the Oklo natural nuclear reactor site in Gabon, Africa, and their atomic weights in those materials are not included in the determination of the standard atomic weight. For elements in category 3 and 4, the Commission uses the footnote " $m$ " to identify those for which the standard atomic weight and its associated uncertainty in commercially available material do not include variations due to undisclosed or inadvertent isotopic fractionation. Changes to the standard-atomic-weight values and uncertainties result from improved measurements of the atomic masses, and these changes primarily affect category 2 elements.”

As discussed in the 2011 TSAW [7],

"If the standard-atomic-weight uncertainty for an element has been expanded because of reported variation in isotopic composition in normal materials, but the Commission has not assigned an interval, a footnote " $\mathrm{r}$ " is retained in the Table of Standard Atomic Weights until the Commission completes an evaluation and determines lower and upper bounds from published data.”

\section{Past footnotes and annotations of atomic-weight tables}

The identification of variations in abundances of stable isotopes upon atomic weights was recognized at the September 1951 meeting of the Commission on Atomic Weights of the International Union of Pure and Applied Chemistry (IUPAC), and an atomic-weight "range” was 
assigned to an element —an assignment of $32.066 \pm 0.003$ to sulfur [8]. In the 1951-1955 TSAWs [8-10], only two footnotes appeared:

a A value given in brackets denotes the mass number of the isotope of the most stable known isotope.

b Because of natural variations in the relative abundances of the isotopes of sulfur the atomic weight of this element has a range of \pm 0.003 .

As opposed to the last two decades, for elements having no stable isotopes that are given footnote asterisk $(*)$ today and that have no characteristic terrestrial isotopic composition, the Commission provided the mass number of the most stable isotope, given in brackets, and used footnote "a” for clarification through the 1955 TSAW, after which it was dropped. Footnote "b" about sulfur was an early recognition of non-radiogenic natural isotopic variability of an element from different sources, and after the 1955 TSAW this footnote became "a". With advances in technology, other elements joined sulfur, and by the 1961 TSAW [11] footnotes elucidated natural isotopic variation in hydrogen, boron, carbon, oxygen, silicon, and sulfur. A second footnote, "b”, elaborated experimental uncertainties for five elements; today evaluated uncertainties are provided following $A_{\mathrm{r}}(\mathrm{E})$ values in parentheses (following the last significant digit to which they are attributed) for all elements. The Commission ceased publishing mass numbers of elements having no stable isotopes in its 1961 TSAW [11]. Instead, a separate table for radioactive elements provided selected mass numbers and half-lives. The 1967 TSAW footnotes are [12]:

a Atomic weights so designated are known to be variable because of natural variations in isotopic composition. The observed ranges are:

$\begin{array}{llll}\text { Hydrogen } & \pm 0.00001 & \text { Oxygen } & \pm 0.0001 \\ \text { Boron } & \pm 0.003 & \text { Silicon } & \pm 0.001 \\ \text { Carbon } & \pm 0.00005 & \text { Sulfur } & \pm 0.003\end{array}$

b Atomic weights so designated are believed to have the following experimental uncertainties:

$\begin{array}{llll}\text { Neon } & \pm 0.003 & \text { Copper } & \pm 0.001 \\ \text { Chlorine } & \pm 0.001 & \text { Bromine } & \pm 0.001 \\ \text { Iron } & \pm 0.003 & \text { Silver } & \pm 0.001\end{array}$

The 1969 TSAW [4] was also the first to include a substantial expansion in the number and an extended discussion of footnotes, recognizing their inherent value to users of the TSAW. For the first time, this TSAW pointed out that these footnotes should be included when the TSAW is reprinted in text, reference books, or related publications. The Commission attempted to state atomic-weight values as precisely as possible, but they recognized that [4]

"At the same time, they must be sufficiently imprecise so that all normal specimens fall within the implied tolerance range. In other words, large quantities of available materials should not lie outside the tolerance range. The difficult judgment has to be made when only a small fraction of normal material falls outside the tolerance range. The Commission has decided in such cases not to discard useful accuracy applicable to the great majority of practical conditions, but to exclude from the definition of 'normal', geological oddities."

The footnotes of the 1969 TSAW [4] were: 
a Mononuclidic element.

b Element with one predominant isotope (about 99-100 \% abundance).

c Element for which the atomic weight is based on calibrated measurements.

d Element for which variation in isotopic abundance in terrestrial samples limits the precision of the atomic weight given.

e Element for which users are cautioned against the possibility of large variations in atomic weight due to inadvertent or undisclosed artificial isotopic separation in commercially available materials.

f Most commonly available long-lived isotope; see 'Table of Selected Radioactive isotopes.'

g In some geological specimens this element has a highly anomalous isotopic composition, corresponding to an atomic weight significantly different from that given.

Footnote “a” implied great accuracy of the atomic-weight value. Footnote "b” alerted uses that errors or variations in isotopic abundance measurements will have a rather small effect on the atomic-weight value. Footnote "c" "implied confidence in the reliability of an atomic-weight value through careful physical measurements based on comparisons with synthetic mixture of almost pure isotopes. To this date, the only atomic weight values that in the Commission's judgment qualify for this annotation have been derived from mass spectrometry. [4]" These three footnotes "were later discarded because they had no consequential effect for the user other than was inherent in the tabulated atomic weight and uncertainty values." [2] Footnote " $\mathrm{f}$ " (Most commonly available long-lived isotope) was later discarded because a table of radioactive isotopes was provided. The footnote "g", which indicated some geological specimens could have a highly anomalous isotopic composition and atomic weight, was attached to argon, strontium, lead, and radium. Footnote "g" was attached to strontium because some thirty years before strontium in a rubidium-rich sample was found to have a ${ }^{87} \mathrm{Sr}$ amount fraction of 1 due to decay of ${ }^{87} \mathrm{Rb}$. Three of these footnotes, “d”, “e”, and " $\mathrm{g}$ ”, continue even now, as footnotes " $\mathrm{r}$ ", “ $\mathrm{m}$ ”, and "g", respectively. There were small changes in wording in each of these footnotes since publication of the 1969 TSAW [4], but the intended meaning has remained generally the same.

\subsection{History of footnote " $r$ ", range in isotopic composition of normal material prevents a more precise $A_{r}(E)$ being given}

In the 1969 TSAW [4], only ten elements (hydrogen, lithium, boron, carbon, oxygen, silicon, sulfur, argon, copper, and lead) were assigned footnote “d” (now “ $\mathrm{r}$ ”). By publication of the 2007 TSAW [13], the number of elements had grown to eighteen. The Commission clearly recognized that the number of elements with this footnote would certainly grow as new technology developed, stating [4]:

"Whereas at the present time only a few atomic weights of lighter elements (and those of copper and lead) are qualified by this footnote, one must expect an ever increasing number of atomic weight values to require this annotation. This trend is a natural consequence of greater precision of experimental determination and of progressively increasing information on variable isotopic composition of normal materials. It is most difficult to assess the 
reliability of many atomic weight values in the absence of good information on isotopic variability. The Commission has been conscious of the possible systematic errors that may be involved. Germanium is an example of such an element, which for this or other reasons is likely to need an upward adjustment when better data become available. It is, however, a well-established principle of the Commission to revise values only on the basis of published work that in the judgment of the Commission advances significantly the confidence to be placed in the value.”

Footnote "d" was renamed footnote "w" in the 1975 TSAW [14] and to "r" in the 1981 TSAW [15]. For elements having interval atomic-weight values, footnote " $\mathrm{r}$ " is no longer needed $[6,7,16]$.

\subsection{Normal material}

Today the TSAW informs readers that values apply to "normal" materials. A normal material was defined in the 1969 TSAW [4] and again by Peiser et al. [2], and the definition is provided in section 1. The first use of normal material in a footnote was in the 1971 TSAW [5] for footnote "d":

Element for which known variations in isotopic abundance in terrestrial material prevent a more precise atomic weight being given; $A_{r}(E)$ values should be applicable to any 'normal' material.

\subsection{History of footnote " $\mathrm{m}$ ", Modified isotopic compositions may be found in commercially available material because the material has been subjected to an undisclosed or inadvertent isotopic fractionation}

Since 1969, the Commission has had serious concerns about the potential impact of elements having the possibility of large variations in atomic weight due to inadvertent or undisclosed artificial isotopic separation (fractionation) in commercially available materials (footnote " $\mathrm{e}$ " in the 1969 TSAW [4], and now footnote " $m$ ") although it only applied in 1969 to three elements (lithium, boron, and uranium). The Commission wrote [4]:

"The seriousness of the hazard must be stressed for users of atomic weight values qualified by footnote e. The atomic weight values given in the Table are neither adjusted nor is their implied accuracy limited to include significant human interference in isotopic abundance. Suppliers of an otherwise well characterized chemical containing as a major constituent one of the elements so annotated should consider the desirability of either supplying the relevant atomic weight value or certifying the virgin source of the raw material plus a statement of the extent to which subsequent processing is expected to have changed the isotopic abundance. . .

It can be foreseen, however, that advancing technology will gradually introduce such complications for other elements. Time is not far off, for example, when fission products will be introduced into normal trade. The Commission on Atomic Weights clearly must keep this situation under careful and continuing review.” 
Footnote "e" was renamed footnote " $y$ " in the 1975 TSAW [14] and to " $m$ " in the 1981 TSAW [15]. Footnote " $\mathrm{m}$ " has been retained for the twelve elements having interval atomic-weight values to alert the reader that commercial materials can be found that have undergone undisclosed or inadvertent isotopic fractionation [6].

\subsection{History of footnote "g", geological materials are known in which the element has an isotopic composition outside the limits for normal material}

The first appearance of footnote " $g$ " to identify an element that has an isotopic composition outside the limits of normal materials is found in the 1969 TSAW [4]. In the 1975 TSAW [14] footnote "g" was renamed footnote "x". Subsequently, in the 1981 TSAW [15] it was renamed footnote "g" again. In the 1970s, this footnote was used to account for specimens from the natural nuclear chain reactor discovered in 1972 at the Oklo quarry in Gabon, Africa and first discussed in the 1973 TSAW[17]. Further investigation of Oklo specimens has now confirmed the occurrence of anomalous antimony, cadmium, cerium, dysprosium, erbium, europium, gadolinium, krypton, molybdenum, neodymium, palladium, rubidium, ruthenium, samarium, silver, tellurium, tin, uranium, xenon, ytterbium, and zirconium $[2,18,19]$. In the 1985 TSAW [20], footnote "g" was attached to boron to account for seawater boron because it was considered unlikely that it would become a source of commercial boron. With the introduction of interval standard atomic-weight values in 2009, footnote "g" usually is no longer needed for these interval elements $[6,7,16]$.

\subsection{History of footnote asterisk (*), element has no stable isotopes}

The footnote asterisk $(*)$ has been modified from its first use in the 1983 TSAW [21] by combining it with other footnotes over the years. Four of the seven footnotes in the 1983 TSAW [21] are:

A Radioactive element that lacks a characteristic terrestrial isotopic composition. One or more well-known isotopes are given in Table 3 with the appropriate mass and half-life.

$\mathrm{X}$ Thorium has a well defined (mononuclidic) composition in minerals with only rare exceptions. In certain places, however (most notably in ocean water), measurable quantities of ${ }^{230}$ Th (Ionium) can be found.

Y Uranium is the only element with no stable isotopes but which has a characteristic terrestrial composition of long-lived isotopes such that a meaningful atomic weight can be given for natural samples.

* Element has no stable isotopes.

In the 1985 TSAW [20], footnotes " $\mathrm{X}$ " and " $Y$ " were discarded, and one new footnote was added:

Z An element, without stable nuclide(s), exhibiting a range of characteristic terrestrial compositions of long-lived radionuclide(s) such that a meaningful atomic weight can be given.

In the 1989 TSAW [22] footnotes “A”, “Z”, and asterisk (*) were combined to the following: 
* Element has no stable nuclides. One or more well-known isotopes are given in Table 3 with the appropriate relative atomic mass and half-life. However, three such elements (Th, $\mathrm{Pa}$ and $\mathrm{U}$ ) do have a characteristic terrestrial isotopic composition, and for these an atomic weight is tabulated.

Table 3 was titled “Relative Atomic Masses and Half-Lives of selected Radionuclides”. In the 2011 TSAW [7], the footnote text was amended to account for the recognition that bismuth is a radioactive element, having no stable isotopes:

* Element has no stable isotopes. One or more representative isotopes are given in Table 3 with the appropriate relative atomic mass and half-life. However, four such elements (Bi, Th, $\mathrm{Pa}$, and $\mathrm{U}$ ) do have a characteristic terrestrial isotopic composition, and for these elements, standard atomic weights are tabulated.

\subsection{History of footnote dagger $(\dagger)$, commercially available Li materials have atomic weights that range between 6.94 and 6.99}

The exceptional situation with respect to the wide variation in isotopic compositions of lithium reagents due to undisclosed artificial isotopic separation [23,24] was addressed by including a special footnote, dagger $\left({ }^{\dagger}\right)$, in the 1993 TSAW [25] to publicize the large variations of $A_{\mathrm{r}}(\mathrm{Li})$ in commercial products. This footnote is:

$+\quad$ Commercially available Li materials have atomic weights that range between 6.94 and 6.99; if a more accurate value is required, it must be determined for the specific material.

The text was updated in the 1997 TSAW [26] to:

$+\quad$ Commercially available Li materials have atomic weights that range between 6.939 and 6.996; if a more accurate value is required, it must be determined for the specific material.

In the 2009 TSAW [16], the lithium footnote was dropped because lithium was assigned an interval atomic-weight value, [6.938, 6.997].

\section{Current footnotes and annotations of the Table of Isotopic Compositions}

The first "Table of Isotopic Compositions of the Elements as Determined by Mass Spectrometry" was published as a part of the 1975 TSAW [14]. It contained "Notes" in column 5 as well as footnotes. The intent was that symbols in the "Notes" column were correlated to footnotes of TSAW. A similarly-named table was published in the 1977 TSAW [27]. Beginning with the 1981 Commission meeting, this table was published at about 6-year intervals as a separate publication, the Table of Isotopic Compositions of the Elements (TICE) [28]; for example, in 1983 [29], in 1989 [30], in 1997 [31], in 2001 [32], in 2009 [33], and most recently in 2013 [34]. With the 1981 TICE, the title of column 5 was changed to "Annotations", but its function remained the same. Each TICE contained a variety of footnotes that typically applied to entries in columns other than column 5 , and these footnotes commonly were retained only for a single TICE. Commonly the TICE footnotes applied to isotope-amount fractions of the best 
measurement column (column 6) or to representative isotopic abundances (column 9). It is not the intent of this article to discuss the footnotes in each TICE. The latest (2013) TICE annotations [34] have been harmonized with latest TSAW (2013) footnotes [6] shown in section 2:

g geologically exceptional specimens are known in which the element has an isotopic composition outside the reported interval.

m modified isotopic compositions may be found in commercially available material that has been subjected to an undisclosed or inadvertent isotope fractionation. Substantial deviations from the listed isotopic compositions can occur (refers to Column 9).

$\mathrm{r}$ range in isotopic composition of normal terrestrial material prevents more precise values (for Column 9) to be given. The tabulated values should be applicable to any normal material.

There is no need for footnote asterisk $\left(^{*}\right)$ to identify radioactive elements with no stable isotopes. Instead, data columns in TICE are left blank for such elements. Data are provided for bismuth, thorium, protactinium, and uranium because these elements have characteristic terrestrial isotopic compositions and standard atomic-weight values can be determined for them.

\section{Past notes and annotations of isotopic composition tables}

The notes of the 1975 TICE, found in column 5, are:

$\mathrm{R}$ Range corresponds to that of known natural variations.

D Range corresponds to differences between published values not supported by established natural variations.

U Range falls within the uncertainties quoted in column 4.

G Element is known to have a highly anomalous composition in certain, specific, geological specimens.

$\mathrm{X}$ Data from one measurement is available and any possible variations are not known.

There was not a one-to-one correlation between the notes of the 1975 TICE and footnotes in the 1975 TSAW [14], which had the TSAW footnotes "w”, “ $x$ ”, " $y$ ”, and " $z$ " as discussed in section 3. However, "R" and " $w$ " were similar, and "G" and " $y$ " were similar.

In 1981, the Commission introduced a minimum and coherent set of single-symbol footnotes and annotations, which were harmonized between the Tables of Standard Atomic Weights [15] and the Table of Isotopic Compositions [28], respectively. The annotations are:

$\mathrm{R}$ Range in isotopic composition in normal terrestrial material is responsible for part, or all, of the difference between limits of reported values.

G Geologically exceptional specimens are known in which the element has an isotopic composition outside the limits of reported values.

O One measurement only provides the available data. 
M Modified isotopic compositions may be found in commercial material that will fall outside the limits listed, because the material has, either deliberately or inadvertently (see notes), been subjected to isotopic separation.

$\mathrm{L} \quad$ Longest half-life isotope mass is chosen for the tabulated $A_{\mathrm{r}}$ value.

The annotations are uppercase for the 1981 TICE [28] to maintain some distinction between them and the footnotes of the 1981 TSAW [15], whose footnotes are lowercase.

In the 1989 TICE [30], the Commission reduced the number of annotations to three ("g", " $m$ ", and "r") and made them lowercase to harmonize them with the 1989 TSAW [22] footnotes. These three have been retained to the present day. Although the wording has changed slightly during the decades, the intent of the annotations has remained the same.

\section{Membership of sponsoring bodies}

Membership of the IUPAC Inorganic Chemistry Division Committee for the period 2014-2015 was as follows:

President: J. Reedijk (Netherlands); Secretary: M. Leskelä (Finland); Vice President: L. R. Öhrström (Sweden); Past President: R. D. Loss; Titular Members: T. Ding (China); M. Drábik (Slovakia); E. Y. Tshuva (Israel); D. Rabinovich (USA); T. Walczyk (Republic of Singapore); M. E. Wieser (Canada); Associate Members: J. Buchweishaija (Tanzania); J. Garcia-Martinez (Spain); P. Karen (Norway); A. Kilic (Turkey); K. Sakai (Japan); R.-N. Vannier (France); National Representatives: F. Abdul Aziz (Malaysia); L. Armelao (Italy); A. Badshah (Pakistan); V. Chandrasekhar (India); J. Galamba Correia (Portugal); S. Kalmykov (Russia); L. Meesuk (Thailand); S. Mathur (Germany); B. Prugovecki (Croatia); N. Trendafilova (Bulgaria).

Membership of the IUPAC Commission on Isotopic Abundances and Atomic Weights for the period 2014-2015 was as follows:

Chair: J. Meija (Canada); Secretary: T. Prohaska (Austria); Titular Members: W. A. Brand (Germany); M. Gröning (Austria); R. Schönberg (Germany); X.-K. Zhu (China); Associate Members: T. Hirata (Japan); J. Irrgeher (Austria); J. Vogl (Germany); National Representative: T. B. Coplen (USA).

Acknowledgments: Comments by H. A. J. Meijer (University of Groningen, Groningen, The Netherlands), J. Meija (National Research Council Canada, Ottawa, Canada), and J.K. Böhlke (U.S. Geological Survey, Reston, Virginia, USA) improved this work and are appreciated. The support of the U.S. Geological Survey National Research Program made this report possible. The following IUPAC project contributed to this Technical Report: 2015-030-2-200.

\section{References}

[1] N. E. Holden, Pure Appl. Chem. 52, 2349 (1980). 
[2] Element-by-element review: H. S. Peiser, N. E. Holden, P. De Bièvre, I. L. Barnes, R. Hagemann, J. R. de Laeter, T. J. Murphy, E. Roth, M. Shima, H. G. Thode. Pure Appl. Chem. 56, 695 (1984).

[3] J. R. de Laeter, J. K. Böhlke, P. De Bièvre, H. Hidaka, H. S. Peiser, K. J. R. Rosman, P. D. P. Taylor. Pure Appl. Chem. 75, 683 (2003).

[4] “Atomic weights of the elements 1969”, Pure Appl. Chem. 21, 91 (1970).

[5] “Atomic weights of the elements 1971”, Pure Appl. Chem. 30, 637 (1972).

[6] J. Meija, T. B. Coplen, M. Berglund, W. A. Brand, P. De Bièvre, M. Gröning, N. E. Holden, J. Irrgeher, R. D. Loss, T. Walczyk, T. Prohaska, Atomic Weights of the Elements 2013, Pure Appl. Chem., 2016 in press. doi: 10.1515/pac-2015-0305

[7] M. E. Wieser, N. Holden, T. B. Coplen, J. K. Böhlke, M. Berglund, W. A. Brand, P. De Bièvre, M. Gröning, R. D. Loss, J. Meija, T. Hirata, T. Prohaska, R. Schoenberg, G. O’Connor, T. Walczyk, S. Yoneda, X.-K. Zhu. Pure Appl. Chem. 85, 1047 (2013).

[8] E. Wichers. J. Am. Chem. Soc. 74, 2447 (1952).

[9] E. Wichers. J. Am. Chem. Soc. 76, 2033 (1954).

[10] E. Wichers. J. Am. Chem. Soc. 78, 3235 (1956).

[11] A. E. Cameron, E. Wichers. J. Am. Chem. Soc. 84, 4175 (1962).

[12] “Atomic weights of the elements 1967”, Pure Appl. Chem. 18, 569 (1969).

[13] M. E. Wieser, M. Berglund. Pure Appl. Chem. 81, 2131 (2009).

[14] “Atomic weights of the elements 1975”, Pure Appl. Chem. 47, 75 (1976).

[15] N. E. Holden, R. L. Martin. Pure Appl. Chem. 55, 1101 (1983).

[16] M. E. Wieser, T. B. Coplen. Pure Appl. Chem. 83, 359 (2011).

[17] “Atomic weights of the elements 1973”, Pure Appl. Chem. 37, 589 (1974).

[18] H. Hidaka, P. Holliger, A. Masuda. Earth Planet. Sci. Lett. 114, 391 (1993).

[19] H. Hidaka, P. Holliger. Geochim. Cosmochim. Acta 62 (1), 89 (1998).

[20] “Atomic weights of the elements 1985”, Pure Appl. Chem. 58, 1677 (1986).

[21] N. E. Holden, R. L. Martin. Pure Appl. Chem. 56, 653 (1984).

[22] “Atomic weights of the elements 1989”, Pure Appl. Chem. 63, 975 (1991).

[23] N. E. Holden, Chem. Intern. 32 (1), 12 (January-February 2010).

[24] H. P. Qi, T. B. Coplen, Q. Zh. Wang, Y. H. Whang. Anal. Chem. 69, 4076 (1997).

[25] “Atomic weights of the elements 1993”, Pure Appl. Chem. 66, 2423 (1994).

[26] R. D. Vocke, Jr. Pure Appl. Chem. 71, 1593 (1999).

[27] N. E. Holden. Pure Appl. Chem. 51, 405 (1979).

[28] N. E. Holden, R. L. Martin, I. L. Barnes. Pure Appl. Chem. 55, 1119 (1983).

[29] N. E. Holden, R. L. Martin, I. L. Barnes. Pure Appl. Chem. 56, 675 (1984).

[30] “Isotopic compositions of the elements 1989”, Pure Appl. Chem. 63, 991 (1991).

[31] K. J. R. Rosman, P. D. P. Taylor. Pure Appl. Chem. 70, 217 (1998).

[32] J. K. Böhlke , J. R. de Laeter, P. De Bièvre, H. Hidaka, H. S. Peiser, K. J. R. Rosman, P. D. P. Taylor. J. Phys. Chem. Ref. Data 34, 57 (2005).

[33] M. Berglund, M. E. Wieser. Pure Appl. Chem. 83, 397 (2011). 
[34] J. Meija, T. B. Coplen, M. Berglund, W. A. Brand, P. De Bièvre, M. Gröning, N. E. Holden, J. Irrgeher, R. D. Loss, T. Walczyk, T. Prohaska, Isotopic Compositions of the Elements 2013, Pure Appl. Chem., 2016 in press. doi: 10.1515/pac-2015-0503 